\title{
A Study of the Effect of Rapid Fluid Intake on Quality of the Bone Scintigraphy Image
}

Nader Mafi ${ }^{*}$ and Nicholas Dodd

Auckland Radiology Group, Auckland, New Zealand

*Corresponding author: Nader (Nick) Mafi, Auckland Radiology Group, Auckland, New Zealand, E-mail: nick.mafi@globalmedicalsolutions.com

Received date: October 06, 2018; Accepted date: October 20, 2018; Published date: October 29, 2018

Copyright: @2018 Mafi N, et al. This is an open-access article distributed under the terms of the Creative Commons Attribution License, which permits unrestricted use, distribution, and reproduction in any medium, provided the original author and source are credited.

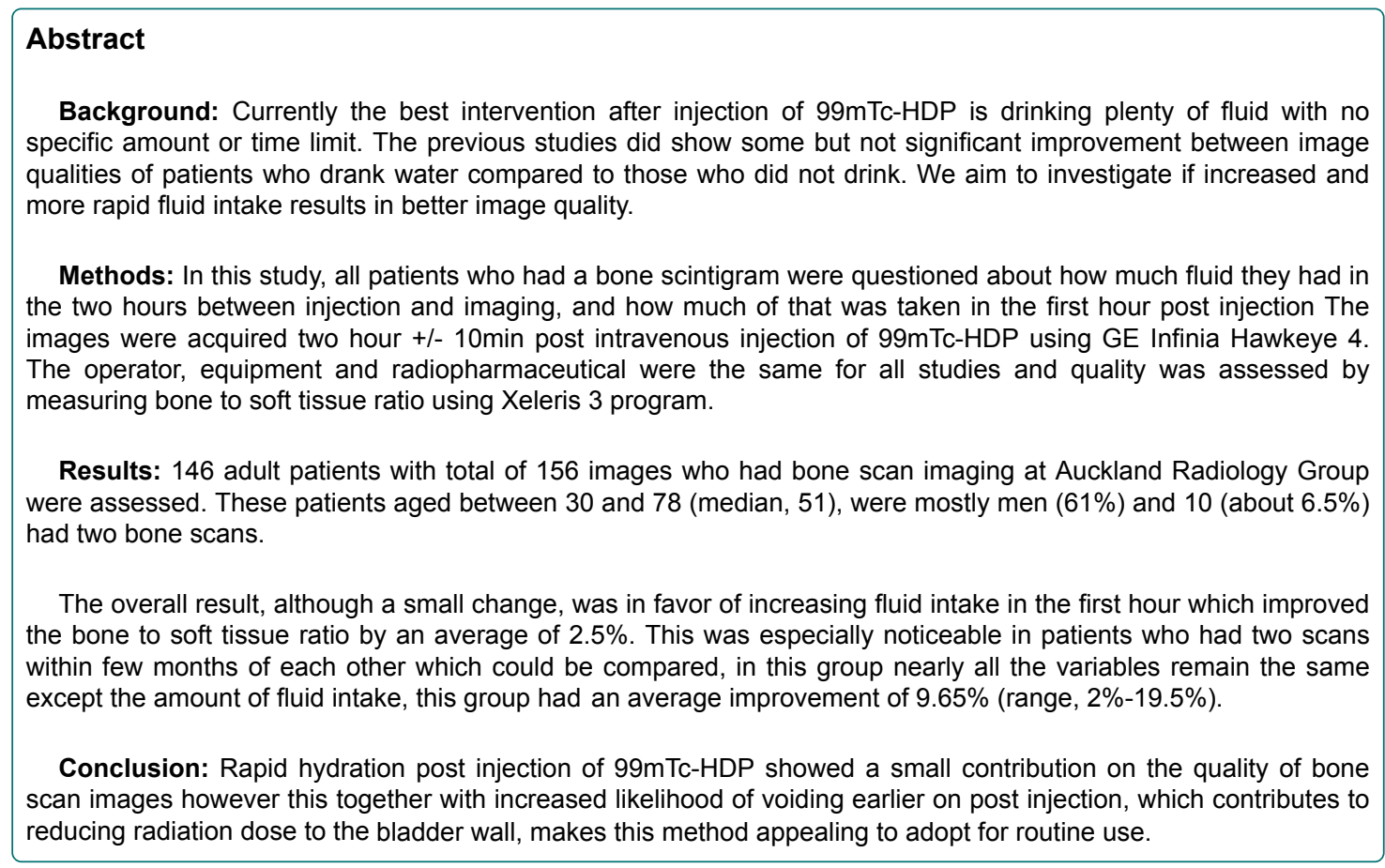

Keywords: Bone scintigraphy; 99mTc-HDP; Rapid fluid intake

\section{Introduction}

Bone scintigraphy using $99 \mathrm{mTc}$ is among the most commonly performed Nuclear Medicine procedures. Although it has low specificity, it is its high sensitivity that makes it highly useful for screening and diagnosis of many conditions that are not easily visualized by other radiological modalities [1].

There are several radiopharmaceutical bone agents with various excipients available to use for bone scan. Generally depending on the bone agent, rate of blood flow and new bone formation [2], approximately half the administered dose accumulates in the bone between 2-6 hours being absorbed to the osseous mineral phase with minimal attachment to the organic phase. In the published quality guidelines for nuclear medicine in skeletal scintigraphy using $99 \mathrm{mTc}$ MDP, or similar product, patient is advised to increase fluid intake and voiding more often $[3,4]$.

In this study we used HDP, this product is rapidly taken up in the skeleton, and has a kidney clearance rate of approximately $30 \%$ in first hour, $48 \%$ by two hours and $60 \%$ within 6 hours post injection. The reason for hydration is to increase the renal excretion of the unbound bi- and diphosphonate which should ultimately results in superior bone-to-soft tissue (B: ST) ratios and better quality images as well as reducing the dose to bladder.

Although here is no clinical study to support this hypothesis. Further more, Cronhjort et al. also mentioned that the advantage of fluid intake on bone scan's image quality is not proven and cannot disregard having a possible undesirable effect. Hence, it currently remains unclear that hydration could have any effect on the quality of the image.

Study of the medical literature [MEDLINE till 2013] found three clinical studies regarding fluid uptake and image quality in nuclear medicine bone scan in humans [5-7].

On reviewing their procedure we found that Klemenz [6] hydrated all his subjects with various hydration levels while Cronhjort [5] studied a small number of people (10 volunteers) and in the Stace et al. [7] study there were 200 subjects but with no assessment of post injection fluid intake in their participants as they looked at influence of increase fluid intake prior to radiopharmaceutical injection on image quality although, and there was also inconsistency in their study groups regarding the subject's physical activity levels $[8,9]$. 
The study by Amin [10] during the Holy month of Ramadan in Egypt revealed no significant different in image quality during this month, when patients were not drinking or eating, and non-fasting months.

\section{Methodology}

The study was designed as an audit therefore The Health and Disability Ethics Committees in New Zealand has indicated that submission to HDEC is not required.

146 patients with ordinary day by day movement, for example, strolling or shopping who were referred for bone scan by their medical specialist were chosen, they were all asked to drink plenty of fluid after injection and come back for scan two hours post injection, once they were back they were asked as how much they managed to drink in the two hours and how much of that was in the first hour and according to their response they were categorized as: Group 1 who were properly hydrated by 1 Litre or more fluid in the first hour post injection and group 2 who were hydrated less in the first hour and overall during the two hour period post injection. These groups had similar characteristics (Age, gender, weight, height, BMI, and thigh circumference).

The clinical indications for imaging in vast majority (approximately $85 \%)$ were metastatic and or localized or generalized bone pains. In order to keep the external variables to a minimum we excluded the patients who had elevated serum creatinine, disorder in urine voiding, known impaired kidney function, on long term iron or aluminum base antacid medication, very obese, had prosthesis in the region where the calculation was done, abnormalities in soft tissue specifically in the upper leg regions such as lymphedema, and pediatric groups, and patients who had more than one bone scan within a few months of each other were included where possible.

All imaging studies were performed $(120 \pm 10$ minutes $)$ post injection of $99 \mathrm{mTc}-\mathrm{HDP}$ using GE Infinia Hawkeye 4 gamma camera which was mounted with a low energy high resolution collimator (scan speed, $16 \mathrm{~cm} / \mathrm{min}$; normal FE mode, uniformity map of $7.5 \%$ and peak energy level of 140; matrix, $256 \times 1024$ ), and Xeleris 3 software.

Quality of the images was assessed both visually and mathematically measuring bone to soft tissue ratio similar to the methodology of Amin et al. [11]. The visual evaluation of the image quality included the following semiquantitative criteria: Bone to soft tissue contrast, assessment of one thoracic rib in Both the anterior and posterior images, and $3^{\text {rd }}$ lumbar vertebrae. The assessment scale was base at: Optimal visibility, six points; average or suboptimal, three points; and insufficient, zero points. Scans with 12 to 18 points were assessed as finest, 6 to 11 points were average or suboptimal, and zero to 5 points were poor.

Calculation for the bone to soft tissue was performed base at the counts over an area of the femoral diaphysis by drawing the region-ofinterest (ROI). A ROI had a minimum size of 150 pixels, this was compared with a same sized ROI of the adductor area. Bone to soft tissue ratio was calculated from the mean total number of counts of anterior and posterior views from bone ROI and soft tissue ROI Statistical methods.

All data were recorded in the form of bone counts and the soft tissue counts, and the ratio was calculated. In our opinion the most accurate data were the ones from patients who had two bone scans at which they drank more fluid in the first hour during one study than for their other scan. In these scans the variables remained the same except for degree of hydration.

\section{Results}

The more hydrated group (G1) and other group (G2) comprised 78 patients each ( 47 males and 31 females) with their mean ages $53.6 \pm$ 12.5 and 55.6 years \pm 10.3 respectively. No significant difference was found statistically between the two groups regarding gender, age, height, weight, BMI, thigh boundary and clinical indications.

Assessment of the pelvic region revealed that in $14 / 78$ (approximately 18\%) of patients in G1 urinary bladder were filled, even though they emptied their bladder immediately just prior to the scan compared to 5/78 (approximately) 6\%) in G2. Over all G1 had more frequent voiding almost twice as frequent as in G2.

The overall means of Bone: Soft Tissue ratio of group 1 and group 2 were calculated to 2.75 and 2.72 respectively with only a small statistical difference (P 0.03), overall improvement of $2.5 \%$, however the mean of our 10 patients who had two studies a few months apart indicated greater improvement between G1 and G2, they were found to be 2.79 and 2.56 respectively corresponding to statistical difference of (P 0.229), an improvement of $9.65 \%$.

\section{Discussion}

This study was performed to evaluate change in image quality after more rapid hydration post injection of $99 \mathrm{mTc}-\mathrm{HDP}$. This study found a small increase in measured bone to soft tissue ratio and in subjective image quality after more rapid hydration post IV injection of radiopharmaceutical 99mTc-HDP in bone scintigraphy.

In a search of the literature we couldn't locate any past clinical investigations regarding the negative effect of reduce fluid intake on quality of Tc-99m bone scans images. Cronhjort et al. did two studies one using animals [8] and one with 10 human volunteers [5]. Stace et al. [7] examined the hydration impact before tracer infusion on picture quality and in our opinion their main impediments was the inconsistency in physical activity levels between the compared groups. It is recognized that increase hydration will increase the extracellular fluid volume which result in lower concentration of 99mTc HDP in blood plasma this has a potential to reduced renal and bone extraction rates [8]. Skeletal uptake and renal excretion almost start immediately post injection.

Skeletal uptake accounts for about $50 \%$ of administered dose most of which occurs in first 30 min post injection and renal excretion accounts for $30 \%$ of dose in the first hour post injection. Amin et al. [10] discover that image quality of the bone scintigraphy is not influenced by fluid restriction or increase hydration.

Cronhjort et al. showed in mice [8], followed by in humans [5] that increase or decrease in water intake had no impact in the bone to soft tissue ratio. Klemenz et al. [6] analyzed the effect of low quantity $(0.25$ Litre) with high quantity (1-5 Liter) of water consumption after the injection of Tc-99m MDP on bone scan image quality and found no significant difference between the two groups [11].

Lastly, Stace et al. [7] study concluded that increase in fluid intake prior to tracer injection has minimal effect on the quality of bone scan images, so, as far as we know there is no similar method was used in any other studies to evaluate the effect of rapid/early increase in fluid intake versus other means of hydration post $99 \mathrm{mTc}$ HDP injection on 
the quality of the image in bone scintigraphy in patients with normal day to day activity levels.

It is not fully understood how diphosphonate is clear by kidneys in hydrated adult patients, whoever it appears that approximately $10 \%$ of the injected dose is evident in whole blood and $50 \%$ in the skeletal 30 min after injection. consequently, it would be less likely that a mildly increased in hydration would speed up renal excretion of the tracer to a reasonable level [8] but rapid increase may do. It is also possible that increased hydration may to a certain level, increase the volume of extracellular fluid therefore lead to a higher distribution volume of 99mTc-HDP, like 99mTc-MDP, and result in reduction in plasma levels and consequently lower bone and renal extraction rates $[5,6,9]$.

In addition, evaluation of pelvic bony detail was limited when the urinary bladder was filled as seen in some of our patients $(18 \%$ in group $1 \mathrm{vs} .6 \%$ in group 2) even when they void immediately before the scan. In the other hand the main concern with low fluid intake is that it will increase radiation dose to the bladder wall as well as gonads.

We believe for adult patients with normal day to day activity, rapid hydration post injection of $99 \mathrm{mTc}-\mathrm{HDP}$ has a small but worthwhile influence on bone to soft tissue ratios/image quality especially when images are acquired approximately two hours post injection of $99 \mathrm{mTc}$ HDP. This has the added benefit of increasing voiding frequency and volume, and therefore reduces the dose especially to bladder and to lesser degree to the surrounding organs including gonads.

Hence, the conclusion of our study could be beneficial in instructing all patients to increase fluid intake, particularly in younger men, and women of childbearing age.

\section{Conclusion}

Quantitative evaluation of data gathered from this study indicates that more rapid hydration post injection of 99mTc-HDP improves bone scan image quality both subjectively and by increasing measured bone to soft tissue ratio, for images taken approximately two hours post injection. Although a small improvement this together with increased likelihood of voiding earlier following radiopharmaceutical injection, which contributes to reducing dose to the bladder wall, makes increased and earlier fluid intake appealing to use on a routine basis.

\section{References}

1. Genant HK, Bautovich GJ, Singh M, Lathrop KA, Harper PV (1974) Bone-seeking radionuclides: An in vivo study of factors affecting skeletal uptake. Radiology 113: 373-382.

2. Brenner AI, Koshy J, Morey J, Lin C, DiPoce J (2012) The bone scan. Seminars in Nuclear Medicine 42: 11-26.

3. BNMS clinical guidelines (2001) $99 \mathrm{mTc}$ diphosphonate bone imaging for metastases. British Nuclear Medicine Society.

4. Donohoe KJ, Brown ML, Collier BD, Carretta RF, Henkin RE (2003) Procedure guideline for bone scintigraphy. Society of Nuclear Medicine. Version 3.0, 205-209.

5. Cronhjort M, Johansson L, Jacobsson H (1997) Hydration does not influence the image quality in bone scintigraphy: An investigation using 99Tcm-HDP. Nuclear Medicine Communication 18: 932-936.

6. Klemenz B, Katzwinkel J, Kaiser KP, Wieler HJ (1999) The influence of differences in hydration on bone-to-soft tissue ratios and image quality in bone scintigraphy. Clinical Nuclear Medicine 24: 483-487.

7. Stace SM, Huggett SM, Denton BK, Harries AM (2003) The value of patient hydration prior to bone scintigraphy. J Diagn Radiog Imag 5: 13-8.

8. Cronhjort M, Schnell PO, Jacobsson H (1994) Disturbances of fluid balance reduce the image quality of bone scintigraphy. Experimental studies in mice. Nuclear Medicine Communication 15: 469-474.

9. O'Connor MK, Brown ML, Hung JC, Hayostek RJ (1991) The art of bone scintigraphy- technical aspects. Journal of Nuclear Medicine 32: 2332-2341.

10. Amin A, Amin M, Nawar A (2013) Does fluid restriction affect the images quality of skeletal scintigraphy? Iran J Nucl Med 21: 77-80.

11. Technescan HDP [Pakage insert] (2014) Westerduinweg, Mallinkrodt Medical B.V 